MITSUBISHI ELECTRIC RESEARCH LABORATORIES

http://www.merl.com

\title{
Model Adjustable Predictive Control with Stability Guarantees
}

\author{
Di Cairano, S.
}

TR2015-059 July 2015

\begin{abstract}
For stabilizing model predictive control adjusting the prediction model requires the adjustment of the terminal set and terminal cost. However, the conventional methods to design these are not practical, and often impossible, to implement in microcontrollers. In this paper, we pre-compute the terminal cost and terminal set in a form that allows to adjust them with minimal computational effort, following an adjustment of the prediction model. For unconstrained systems, a terminal cost and terminal controller are designed based on parameter-dependent Lyapunov functions. For constrained systems, the terminal function is also used to derive a robust polyhedral terminal set. We prove that the proposed method guarantees the existence of a terminal set with non-empty interior, and asymptotic stability of the closed-loop system.
\end{abstract}

2015 American Control Conference (ACC)

This work may not be copied or reproduced in whole or in part for any commercial purpose. Permission to copy in whole or in part without payment of fee is granted for nonprofit educational and research purposes provided that all such whole or partial copies include the following: a notice that such copying is by permission of Mitsubishi Electric Research Laboratories, Inc.; an acknowledgment of the authors and individual contributions to the work; and all applicable portions of the copyright notice. Copying, reproduction, or republishing for any other purpose shall require a license with payment of fee to Mitsubishi Electric Research Laboratories, Inc. All rights reserved. 



\title{
Model Adjustable Predictive Control with Stability Guarantees
}

\author{
Stefano Di Cairano
}

\begin{abstract}
For stabilizing model predictive control, adjusting the prediction model requires the adjustment of the terminal set and terminal cost. However, the conventional methods to design these are not practical, and often impossible, to implement in microcontrollers. In this paper, we pre-compute the terminal cost and terminal set in a form that allows to adjust them with minimal computational effort, following an adjustment of the prediction model. For unconstrained systems, a terminal cost and terminal controller are designed based on parameterdependent Lyapunov functions. For constrained systems, the parameter-dependent Lyapunov function is also used to derive a polyhedral terminal set. We prove that the proposed method guarantees the existence of a terminal set with non-empty interior, and asymptotic stability of the closed-loop system.
\end{abstract}

\section{INTRODUCTION}

While originally developed for chemical and process control, model predictive control (MPC) is now being explored for applications in several other domains such as automotive, aerospace, and manufacturing [1], [2]. These applications impose new challenges and require new developments, such as low complexity optimization algorithms [3]-[6], and calibration methods [7], [8]. In particular, low complexity optimization algorithms [3]-[6] have been motivated by the fact that several of these recent applications exploit microcontrollers that have limited computing power, and where the code has to undergo extensive certification. Thus, the deployed control algorithms should be kept as simple as possible.

In applications where the controller is deployed to hundreds or even thousands of instances of a plant, a significant plant-to-plant variability can be exhibited by some parameters, as well as aging effects, and as a consequence the MPC prediction model may need to be adjusted after the controller has been deployed in the microcontroller. However, when the MPC closed-loop stability is guaranteed by appropriately designed terminal cost and terminal set [9], if the prediction model is adjusted but the terminal cost and terminal set are not, the stability of the closed-loop may be lost. The (re)design of the terminal cost and the terminal set require the solution of LMIs or Riccati equations, and the computation of constrained positive invariant sets. The corresponding computational procedures results in code that is complex to verify, and often has too large computational requirements for production microcontrollers. For similar reasons, a-priori robust MPC based on LMI [10], [11] may not be viable in these applications.

S. Di Cairano is with Mitsubishi Electric Research Laboratories, Cambridge, MA, email: dicairano@ieee.org
In this paper we propose a Model Adjustable Predictive Control (MAPC) algorithm which guarantees with minimal computational effort that stability is preserved after a prediction model adjustment. By designing a parameterdependent terminal cost and a corresponding terminal set, after the MAPC controller has been deployed, the plant can be identified, and the terminal cost and terminal set for guaranteeing the closed-loop stability of the plant controlled by MAPC can be adjusted with a negligible computational cost, i.e., with few simple operations.

We assume that the possible instances of the plant can be obtained as convex combinations of vertex systems. For the case of unconstrained systems, we compute the terminal cost as a function of the convex combination vector of the vertex systems in the form of a parameter-dependent Lyapunov function ( $\mathrm{pLF}$ ). Parameter-dependent Lyapunov functions have been introduced for analysis and robust control design for uncertain linear systems in [12], and have been extended to the control of linear parameter-varying (LPV) systems in [13], [14]. These results are used to design robust MPC [11] and MPC for LPV systems [15], [16], which require the online solution of LMIs. However, the limited capabilities of the microcontroller and the complexity of the LMI solver code makes these methods often impossible to apply in aerospace, automotive, or manufacturing.

In this paper, we do not seek a robust MPC controller for an LPV system, but rather for the possibility of adjusting an MPC design to maintain stability after the prediction model is modified. Thus, starting from [13] we compute before deployment a pLF and a corresponding parameter-dependent controller, so that, when the convex combination vector is estimated after deployment, the terminal cost can be immediately adjusted with minimal computational effort. Since we are not trying to enforce robustness but only adjustability, the actual controller only solves quadratic programs, for which the solvers are much simpler and faster [4], [5].

For the case of constrained MAPC a terminal set is also obtained as the robust maximum constraint admissible set of the vertex systems in closed-loop with the components of the parameter-dependent control law. We show that for the specific parameter-dependent controller obtained together with the pLF, the maximal constraint admissible set has nonempty interior, is polyhedral, and finitely determined.

The rest of the paper is structured as follows. In Section II we describe the system model and the problem addressed in this paper. In Section III we describe the computation of the terminal cost and of a corresponding auxiliary control 
law by parameter-dependent Lyapunov functions, and we establish the stability of unconstrained MAPC. In Section IV we discuss the terminal set design, prove its existence and properties, and establish the stability of constrained MAPC. In Section V we present some numerical examples of MAPC. Finally, in Section VI we summarize the results and discuss the future research directions towards an adaptive MPC framework.

Notation: $\mathbb{R}, \mathbb{R}_{0+}, \mathbb{R}_{+}$are the sets of real, nonnegative real, positive real numbers, and $\mathbb{Z}, \mathbb{Z}_{0+}, \mathbb{Z}_{+}$are the sets of integer, nonnegative integer, positive integer numbers. For intervals of numbers, we use the notation $\mathbb{Z}_{[a, b)}=\{z \in \mathbb{Z}$ : $a \leq z<b\} . \operatorname{co}\{\mathcal{X}\}$ denotes the convex hull of the set $\mathcal{X}$. For vectors, inequalities are intended componentwise, while for matrices indicate (semi)definiteness. By $[x]_{i}$ we denote the $i$-th component of vector $x$, and by $I$ and 0 the identity and the "all-zero" matrices of appropriate dimension. For a discrete time signal $x \in \mathbb{R}^{n}$ with sampling period $T_{s}, x(t)$ is the state at sampling instant $t$, i.e., at time $T_{s} t$. The notation $x_{k \mid t}$ denotes the predicted value of $x$ at sample $t+k$, i.e., $x(t+k)$, based on data at sample $t$, and $x_{0 \mid t}=x(t)$. A function $\alpha: \mathbb{R}_{0+} \rightarrow \mathbb{R}_{0+}$ is of class $\mathcal{K}_{\infty}$ if it is continuous, strictly increasing, $\alpha(0)=0$, and $\lim _{c \rightarrow \infty} \alpha(c)=\infty$

\section{MODELING AND PROBLEM DEFINITION}

First, we review some basic results, see, e.g., [9, App. B].

Definition 1: A set $\mathcal{S} \subset \mathbb{R}^{n}$ is positive invariant (PI) for $x(t+1)=f(x(t)), x \in \mathbb{R}^{n}$, iff $x \in \mathcal{S}$ implies $f(x) \in \mathcal{S} . \square$

Result 1: Given $x(t+1)=f(x(t)), x \in \mathbb{R}^{n}$ a function $\mathcal{V}: \mathbb{R}^{n} \rightarrow \mathbb{R}_{+}$such that there exist a PI set $\mathcal{S} \subseteq \mathbb{R}^{n}$ with $0 \in \mathcal{S}$, and functions $\alpha_{1}, \alpha_{2}, \alpha_{\Delta} \in \mathcal{K}_{\infty}$ such that $\alpha_{1}(\|x\|) \leq \mathcal{V}(x) \leq \alpha_{2}(\|x\|), \mathcal{V}(f(x))-\mathcal{V}(x) \leq-\alpha_{\Delta}(\|x\|)$ for all $x \in \mathcal{S}$, is a Lypaunov function for $f$ in $\mathcal{S}$. If there exists a Lyapunov function for $f$ in $\mathcal{S}$, the origin is asymptotically stable (AS) in $\mathcal{S}$ for $f$.

Consider a discrete-time system sampled with period $T_{s}$,

$$
x(t+1)=\bar{A}(\omega) x(t)+B u(t)
$$

where $x \in \mathbb{R}^{n}, u \in \mathbb{R}^{m}$, and the state update matrix $\bar{A}(\omega)$ depends on a parameter vector $\omega \in \Omega \subset \mathbb{R}^{n_{p}}$. We assume that there exists $\ell \in \mathbb{Z}_{+}$and $A_{i} \in \mathbb{R}^{n \times n}, i \in \mathbb{Z}_{[1, \ell]}$ such that for all $\omega \in \Omega$, there exists $\xi \in \Xi \subset \mathbb{R}^{\ell}, \Xi=\left\{\xi \in \mathbb{R}^{\ell}\right.$ : $\left.0 \leq \xi \leq 1, \sum_{i=1}^{\ell}[\xi]_{i}=1\right\}$, such that $\bar{A}(\omega)=\sum_{i=1}^{\ell}[\xi]_{i} A_{i}$. Thus, system (1) can be represented by

$$
x(t+1)=\sum_{i=1}^{\ell}[\xi]_{i} A_{i} x(t)+B u(t),
$$

where $\xi$ is not known a-priori, but here it is assumed not to change. Due to the form of (2), we call $\xi$ the convex combination vector and $x(t+1)=A_{i} x(t)+B u(t), i \in \mathbb{Z}_{[1, \ell]}$ the vertex systems.

Let the constraints on the system state and input be

$$
x \in \mathcal{X}, \quad u \in \mathcal{U}
$$

where $\mathcal{X} \in \mathbb{R}^{n}, \mathcal{U} \in \mathbb{R}^{m}$ are the sets of admissible states and inputs, respectively.
Consider now the case where $\xi \in \Xi$ is known beforehand. Model predictive control (MPC) uses (2), (3) to setup the finite time optimal control problem

$$
\begin{aligned}
\min _{U_{t}} F\left(x_{N \mid t}\right)+ & \sum_{k=0}^{N-1} L\left(x_{k \mid t}, u_{k \mid t}\right) \\
\text { s.t. } \quad x_{k+1 \mid t}= & \sum_{i=1}^{\ell}[\xi]_{i} A_{i} x_{k \mid t}+B u_{k \mid t} \\
& u \in \mathcal{U} \\
& x \in \mathcal{X} \\
& x_{N \mid t} \in \mathcal{X}_{N} \\
& x_{0 \mid t}=x(t)
\end{aligned}
$$

where $N$ is the prediction horizon, $L: \mathbb{R}^{n} \times \mathbb{R}^{m} \rightarrow \mathbb{R}_{0+}$ is the stage cost, $F: \mathbb{R}^{n} \rightarrow \mathbb{R}_{0+}$ is the terminal cost, $\mathcal{X}_{N}$ is the terminal set and $U_{t}=\left[u_{0 \mid t} \ldots u_{N-1 \mid t}\right]$ is the sequence of control inputs along the prediction horizon. MPC applies to the system the control input $u(t)=u_{0 \mid t}^{*}$, where $U_{t}^{*}=$ $\left[u_{0 \mid t} \ldots u_{N-1 \mid t}\right]$ is the optimizer of (4). In (4), the terminal cost $F$ and the terminal set $\mathcal{X}_{N}$ are designed to guarantee the recursive feasibility and the stability of the closed-loop system, for instance according to the following well known result.

Result 2 ([9]): Let $\kappa(x)$ and $F(x)$ be such that: $(i)$, for all $x \in \mathcal{X}_{N},(4 \mathrm{c}),(4 \mathrm{~d})$ are satisfied for all $x \in$ $\mathcal{X}_{N}$, with $u(x)=\kappa(x) ;(i i), \mathcal{X}_{N}$ is positive invariant for $\sum_{i=1}^{\ell}[\xi]_{i} A_{i} x+B \kappa(x) ;(i i i), F\left(\sum_{i=1}^{\ell}[\xi]_{i} A_{i} x+B \kappa(x)\right)+$ $L(x, \kappa(x))-F(x) \leq 0$ for all $x \in \mathcal{X}_{N}$. Then, the MPC algorithm based on (4) renders the origin of the closed-loop system asymptotically stable.

When $\xi$ in (2) is known a-priori, and the stage cost is quadratic,

$$
L(x, u)=x^{\prime} Q x+u^{\prime} R u, \quad Q, R>0,
$$

a quadratic terminal cost $F(x)=x^{\prime} P x$ and a linear controller $\kappa(x)=K_{f} x$ satisfying the conditions in Result 2 can be computed by solving LMIs or Riccati equations. A polyhedral terminal set $\mathcal{X}_{N}$ is then computed as the maximal constraint admissible set (MCAS) [17] of $x(t+$ 1) $\left.=\sum_{i=1}^{\ell}[\xi]_{i}\left(A_{i}+B K_{f}\right) x(t)\right)$ subject to the constraints $K_{f} x(t) \in \mathcal{U}, x(t) \in \mathcal{X}$.

The computation of LMI requires fairly complicated algorithms, which are unlikely to be deployable in a microcontroller, because they require several complex procedures of linear algebra and large and complex code. Solving Riccati equations may be more manageable, although it still requires more code and more computations. If the terminal cost is adjusted online, even by Riccati equations, the MCAS needs to be adjusted consequently, which once again requires further code and more operations. To avoid additional operations and complexity in the control algorithm, here we aim at solving the following.

Problem 1: Given (2), (3) and (5), compute

(i) a parameter-dependent the terminal cost

$$
F(x, \xi)=x^{\prime} \mathcal{P}(\xi) x
$$


where for any given $\xi \in \Xi, \mathcal{P}(\xi) \in \mathbb{R}^{n \times n}, \mathcal{P}(\xi)>0$, (ii) a terminal set

$$
\mathcal{X}_{N}=\left\{x \in \mathbb{R}^{n}: M_{N} x \leq L_{N}\right\} \subseteq \mathcal{X}
$$

where $M_{N} \in \mathbb{R}^{q \times n}, L_{N} \in \mathbb{R}^{q}, q \in \mathbb{Z}_{+}$,

such that, given any $\xi \in \Xi$, the origin of the closed-loop of (2) with the MAPC controller that at any step solves (4) with (5), (6), (7), and applies $u(t)=u_{0 \mid t}^{*}$ is asymptotically stable, and the constraints in (3) are enforced.

The rationale for Problem 1 is that the terminal cost (6) and terminal set (7) are designed offline and deployed to the controller. When the value of the plant parameter vector $\omega \in \Omega$ is properly estimated, together with the (or $a$ ) corresponding value for $\xi \in \Xi$, the stabilizing terminal cost and terminal set are adjusted with simple computations, and no algorithm other than QP solver for (4)-(7) is needed.

Remark 1: It can be noted that it in Problem 1 the terminal set is not function of $\xi$, and hence we search for a set that is a valid terminal set for all $\xi \in \Xi$. As it will be shown later, for the approach developed here, such set always exists and its conservativeness has a limited effect on the MPC algorithm (unless $N$ is very small), because it is used only as the terminal constraint, and because of the receding horizon behavior of the MPC. The computation of a terminal set as a function of the parameter $\xi$ is a challenging problem, left for future research.

Next, we first propose a design for $\mathcal{P}(\xi)$ in (10) based on parameter-dependent Lyapunov functions [12], [13], which, with a trivial choice of $\mathcal{X}_{N}$, solves Problem 1 for unconstrained MAPC, i.e., when $\mathcal{X}=\mathbb{R}^{n}, \mathcal{U}=\mathbb{R}^{m}$. Then, we solve Problem 1 for constrained MAPC, i.e., when $\mathcal{X} \subset \mathbb{R}^{n}$ and/or $\mathcal{U} \subset \mathbb{R}^{m}$, by a proper selection of $\mathcal{X}_{N}$ in (7).

\section{UnCONSTRAined MAPC: TERMINAL COST DESIGN}

Consider the linear parameter varying system

$$
x(t+1)=\sum_{i=1}^{\ell}[\xi(t)]_{i} A_{i} x(t)+B u(t),
$$

where for all $t \in \mathbb{Z}_{+}, \xi(t) \in \Xi$, the parameter-dependent (linear) control law

$$
u=\kappa(\xi) x=\left(\sum_{i=1}^{\ell}[\xi]_{i} K_{i}\right) x,
$$

and the parameter-dependent (quadratic) function

$$
\mathcal{V}(x, \xi)=x^{\prime} \mathcal{P}(\xi) x=x^{\prime}\left(\sum_{i=1}^{\ell}[\xi]_{i} P_{i}\right) x,
$$

where $P_{i}>0, i \in \mathbb{Z}_{[1, \ell]}$.

Definition 2 ([13]): A parameter-dependent Lyapunov function for (8) in closed-loop with (9) is a function (10) such that

$\mathcal{V}(x(t+1), \xi(t+1))-\mathcal{V}(x(t), \xi(t)) \leq 0, \forall \xi(t), \xi(t+1) \in \Xi$,

that holds with equality only for $x=0$.
Next, we exploit Definition 2 to solve $(i)$ in Problem 1. Definition 2 provides a more stringent requirement that what is necessary for solving Problem 1, since in Definition 2, $\xi$ is time varying, while in Problem $1, \xi$ is constant. However, the more stringent requirement is useful for obtaining design procedures, and for proving properties of the MAPC.

In order to obtain a terminal cost for (4)-(7), we modify (11) to enforce (iii) in Result 2, where the terminal control law is (9). This results in

$$
\begin{array}{r}
\left(\sum_{i=1}^{\ell}[\xi]_{i}\left(A_{i}+B K_{i}\right)\right)^{\prime}\left(\sum_{i=1}^{\ell}[\varsigma]_{i} P_{i}\right)\left(\sum_{i=1}^{\ell}[\xi]_{i}\left(A_{i}+B K_{i}\right)\right) \\
+x^{\prime} Q x+\left(\sum_{i=1}^{\ell}[\xi]_{i} K_{i}\right)^{\prime} R\left(\sum_{i=1}^{\ell}[\xi]_{i} K_{i}\right) \\
-\sum_{i=1}^{\ell}[\xi]_{i} P_{i}<0, \quad \forall \xi, \varsigma \in \Xi .
\end{array}
$$

Thus we design (9) and (10) such that (12) is satisfied.

Lemma 1: Let $G_{i}, S_{i} \in \mathbb{R}^{n \times n}, S_{i}>0, i \in \mathbb{Z}_{[1, \ell]}, E_{i} \in$ $\mathbb{R}^{m \times n}$ be such that

$$
\left[\begin{array}{cccc}
G_{i}+G_{i}^{\prime}-S_{i} & \left(A_{i} G_{i}+B E_{i}\right)^{\prime} & E_{i}^{\prime} & G_{i}^{\prime} \\
\left(A_{i} G_{i}+B E_{i}\right) & S_{j} & 0 & 0 \\
E_{i} & 0 & R^{-1} & 0 \\
G_{i} & 0 & 0 & Q^{-1}
\end{array}\right]>0
$$

Then, $G_{i}$ is full rank for $i \in \mathbb{Z}_{[1, \ell]}$ and $P_{i}=S_{i}^{-1}, K_{i}=$ $E_{i} G_{i}^{-1}, i \in \mathbb{Z}_{[1, \ell]}$ satisfy (12).

The proof of Lemma 1 follow the lines of [13] with modifications inspired from [11] nd from [18, Ch.3.4], which deals with the case where $\xi$, $\varsigma$ can only take a subset of the values of the standard basis in $\mathbb{R}^{\ell}$, and hence is not repeated. Note that LMI (12) is a combination of those in [13], [18] since (13) is similar to [13] in terms of allowing for $\xi, \varsigma \in \Xi$, and similar to [13] due to the need of accounting for the MAPC stage cost in the LMI. Note also that (13) is different from the LMIs in [11] because here we aim at obtaining a parameter dependent control law $\kappa(\xi)$, while in [11] a parameter independent control law for robust control is obtained. A similar, yet slightly different, LMI for online computation has been proposed in [15], and in fact it is simple to prove that (13) and the LMI in [15] are equivalent.

Based on Lemma 1 we can design a parameter-dependent terminal cost that, for any given $\xi \in \Xi$, achieves stability of the closed-loop.

Theorem 1: Let (13) be feasible, and consider (2) controlled by the unconstrained MAPC that at every step solves (4)-(7) where $\mathcal{X}=\mathcal{X}_{N}=\mathbb{R}^{n}, \mathcal{U}=\mathbb{R}^{m}, F(x)=$ $\sum_{i=1}^{\ell}[\xi]_{i} P_{i}$, and $P_{i}=S_{i}^{-1}, i \in \mathbb{Z}_{[1, \ell]}$ satisfy (13). Given any $\xi \in \Xi$, the origin of the closed-loop system is asymptotically stable.

Proof: The proof follows the standard lines of the proofs for unconstrained MPC based on terminal cost [9]. 
Let $\xi \in \Xi$ be given and constant. The value function of the MAPC finite horizon optimal control problem for given $\xi, \mathcal{V}_{\mathrm{MPC}}^{(\xi)}(x)$ is lower and upper bounded by class $\mathcal{K}_{\infty}$ functions, $\underline{\alpha}(\|x(t)\|)=\lambda_{\min }(Q)\|x(t)\|^{2}, \bar{\alpha}(\|x(t)\|)=$ $c_{F} F(x(t))$ for some $c_{F} \in \mathbb{R}_{+}$, see [9, Sec. 2.4.5]. Let $U^{*}(t)=\left[u_{0 \mid t}^{*}, \ldots, u_{N-1 \mid t}^{*}\right]$ be the optimal solution of (4) with initial condition $x(t)$, and $\mathcal{V}_{\text {MPC }}^{(\xi)}(x(t))$ be the corresponding value function. At time $t+1$ from $x(t+1)=x_{1 \mid t}$, the solution $\tilde{U}(t)=\left[\tilde{u}_{0 \mid t+1}, \ldots, \tilde{u}_{N-1 \mid t+1}\right]$ where $\tilde{u}_{i \mid t+1}=$ $u_{i+1 \mid t}^{*}$ for $i \in \mathbb{Z}_{[0, N-2]}, \tilde{u}_{N-1 \mid t+1}=\sum_{i=1}^{\ell}[\xi]_{i} K_{i}$, where $K_{i}$ is from (13) and $\xi \in \Xi$ is known, has cost $\tilde{J} \leq$ $\mathcal{V}_{\text {MPC }}^{(\xi)}(x(t))-x(t)^{\prime} Q x(t)$, due to (12). Since $\mathcal{V}_{\text {MPC }}^{(\xi)}(x(t+$ $1) \leq \tilde{J}$, we have $\mathcal{V}_{\mathrm{MPC}}^{(\xi)}(t+1)-\mathcal{V}_{\mathrm{MPC}}^{(\xi)}(t) \leq-x(t) Q x(t) \leq$ $-\lambda_{\min }(Q)\|x(t)\|^{2}=\alpha_{\Delta}(\|x(t)\|), \alpha_{\Delta} \in \mathcal{K}_{\infty}$. Thus, for any given $\xi \in \Xi, \mathcal{V}_{\mathrm{MPC}}^{(\xi)}(x(t))$ is a Lyapunov function for the closed-loop system when $\xi$ is constant, and hence the origin is asymptotically stable.

Theorem 1 guarantees that once MAPC knows the value of $\xi \in \Xi$, the terminal cost is adjusted by simple summations and multiplications by scalars and the resulting closed-loop is asymptotically stable. Indeed, from a theoretical point of view, MAPC has the same guarantees of an MPC where the model is known at design time. However, MAPC allows for the model to be determined after deployment, because the terminal cost can be adjusted to the actual model by computationally simple operations.

Remark 2: In (1) the input-to-state matrix $B$ is independent of the parameter vector $\xi$ and, as a consequence, also in (2) there is only one matrix $B$. Such limitation can be removed, although in order to obtain LMIs, (9) becomes a parameter-independent controller $\kappa(\xi)=K$, while (10) is still parameter-dependent, see, e.g., [11], [12]. Alternative approaches are also possible. For instance, given a system with input-to-state matrix $B_{\omega}(\omega)$, we may obtain an augmented system with constant $B$ by introducing the incremental input form with an artificial input delay.

\section{Constrained MAPC: TERMinAl SET DESign BASED ON PLDI}

Next, we consider the case of constrained MAPC, i.e., $\mathcal{X} \times \mathcal{U} \subset \mathbb{R}^{n} \times \mathbb{R}^{m}$. For constrained MAPC, we need to modify Theorem 1 to guarantee also recursive feasibility of the finite time optimal control problem (4)-(7). For this, we exploit a terminal set, which is PI for the plant in closedloop with an auxiliary control law that generates feasible trajectories from any initial state within the terminal set. Since in MAPC the model is not exactly known at design time, when (13) is feasible, we use the auxiliary control law (9) designed by Lemma 1 to compute a robust PI set that avoids the online recomputation of the terminal set when the system parameters are identified, i.e., after deployment.

\section{A. Existence of a constrained admissible invariant set}

First notice that the trajectories of the LPV system

$$
x(t+1)=\sum_{i=1}^{\ell}[\xi(t)]_{i}\left(A_{i}+B K_{i}\right) x(t)
$$

where $\kappa(\xi)$ designed according to Lemma 1 , and $\xi(t) \in$ $\Xi$, for all $t \in \mathbb{Z}_{0+}$ include those obtained when $\xi(t)=\xi$, constant. For arbitrarily varying $\xi(t) \in \Xi$, the trajectories of (14) are the same as those generated by the pLDI

$$
x(t+1) \in \operatorname{co}\left(\left\{\left(A_{i}+B K_{i}\right) x(t)\right\}_{i=1}^{\ell}\right) .
$$

Techniques for computing PI sets for LPV systems such as (14) are known, see e.g., [19]. Here we want to prove that the maximal PI set for (14) contained in the feasible set $\mathcal{X}^{\infty} \subseteq \overline{\mathcal{X}}$, where $K_{i}, i \in \mathbb{Z}_{[1, \ell]}$ are obtained from (13), $\overline{\mathcal{X}}=\{x \in \mathcal{X}: \kappa(\xi) x \in \mathcal{U}, \forall \xi \in \Xi\}$, exists and has a non-empty interior, i.e., it is not just $\mathcal{X}^{\infty}=\{0\}$, but instead $0 \in \operatorname{int}\left(\mathcal{X}^{\infty}\right)$. In what follows we outline the main steps of the proof, which follows the lines of that in [17] for linear systems, by stating a list of technical lemmas. The proofs are omitted due to limited space.

Given $x(t) \in \overline{\mathcal{X}}$, if $\left(A_{i}+B K_{i}\right) x(t) \in \overline{\mathcal{X}}$ for all $i \in \mathbb{Z}_{[1, \ell]}$, then for any $\xi(t) \in \Xi, x(t+1)$ obtained from (14), is such that $x(t+1) \in \overline{\mathcal{X}}$. Similarly if $\left(A_{j}+B K_{j}\right)\left(A_{i}+B K_{i}\right) x(t) \in$ $\overline{\mathcal{X}}$ for all $i, j \in \mathbb{Z}_{[1, \ell]}$, then for (14), $x(t+2) \in \mathcal{X}$, for all $\xi(t), \xi(t+1) \in \Xi$. Generalizing, if

$$
\prod_{i=1}^{n_{\xi}}\left(A_{[\varpi]_{i}}+B K_{[\varpi]_{i}}\right) x \in \overline{\mathcal{X}}, \forall \varpi \in \Upsilon_{\ell}\left(n_{\xi}\right), \forall n_{\xi} \in \mathbb{Z}_{[1, t]},
$$

where $\Upsilon_{\ell}\left(n_{\xi}\right)=\mathbb{Z}_{[1, \ell]}^{n_{\xi}}$ is the set of sequences of length $n_{\xi}$ of integers in $[1, \ell]$, then for any $\{\xi(h)\}_{h=0}^{t-1} \in \Xi^{t}$, any sequence $\{x(h)\}_{h=1}^{t}$ generated by (14) from $x(0)=x$ is such that $x(h) \in \mathcal{X}$ for all $h \in \mathbb{Z}_{[1, t]}$.

The existence of PI sets for (8) can be argued by (10) designed according to Lemma 1 being a Lyapunov function for (8). Next, we characterize the shape of such PI sets.

Lemma 2: Let $K_{i}, P_{i}, i \in \mathbb{Z}_{[1, \ell]}$ be obtained as in Lemma 1 . For every $\nu \in \mathbb{R}_{0+}$, the set $\mathcal{S}(\nu)=\bigcap_{i=1}^{\ell} \mathcal{S}_{i}(\nu)$, where $\mathcal{S}_{i}(\nu)=\left\{x: \mathcal{V}_{i}(x) \leq \nu\right\}$ and $\mathcal{V}_{i}(x)=x^{\prime} P_{i} x$, is PI for (14).

Lemma 2 identifies a family of PI sets for (14) that is described by the level sets of $\mathcal{V}_{i}(x)=x^{\prime} P_{i} x, i \in \mathbb{Z}_{[1, \ell]}$ and suggests that we should only track the value of the functions $\mathcal{V}_{i}$ along the trajectories obtained by considering all the sequences of the vertex systems of the pLDI (15).

Lemma 3: Consider $x(t+1)=\left(A_{i(t)}+B K_{i(t)}\right) x(t)$, $i(t) \in \mathbb{Z}_{[1, \ell]}$. Given any bounded $\tilde{\mathcal{X}}$, for every $\rho>0$ there exists a finite $\bar{h}(\rho) \in \mathbb{Z}_{+}$such that for al $x(t) \in \tilde{\mathcal{X}}$ and for all sequences $\{i(t+h)\}_{h=0}^{\bar{h}(\rho)}, \mathcal{V}_{j}(x(t+\bar{h}(\rho))) \leq \rho$, for all $j \in \mathbb{Z}_{[1, \ell]}$.

Lemma 4: Consider (14). There exists a finite index $\bar{h} \in$ $\mathbb{Z}_{0+}$ such that if $x(t+h) \in \overline{\mathcal{X}}$ for all $h \in \mathbb{Z}_{[0, \bar{h}]}$, then $x(t+h) \in \overline{\mathcal{X}}$ for all $h \geq 0$.

Theorem 2: For (14) where $K_{i}, i \in \mathbb{Z}_{[1, \ell]}$ are designed based on Lemma 1, there exists a finetely determined constraint admissible set $\mathcal{X}^{\infty} \subseteq \overline{\mathcal{X}}$ which is positive invariant, and maximal. Furthermore, if $\overline{\mathcal{X}}$ is a polytope, then such constraint admissible set is a polytope.

Proof: Consider $\bar{h}$ from Lemma 4, and the set $\mathcal{X}^{\infty}=$ $\{x \in \overline{\mathcal{X}}:$ (16) holds for $t=\bar{h}\}$. Thus, all the trajectories 
of (14) such that $x(0)=x$, satisfy $x(t) \in \overline{\mathcal{X}}$ for $t \in \mathbb{Z}_{[0, \bar{h}]}$. By Lemma 4 , if $x(t) \in \overline{\mathcal{X}}$ for all $t \in \mathbb{Z}_{[0, \bar{h}]}, x(t) \in \mathcal{X}$ for all $t \in \mathbb{R}_{0+}$. Positive invariance follows directly from the definition of constraint admissible set [17]. As in [17], maximality follows from $\mathcal{X}^{\infty}=\{x \in \overline{\mathcal{X}}: x(0)=x \Longrightarrow$ $x(t) \in \overline{\mathcal{X}}, \forall t \in[0, \bar{h}]\}$, and from the definition of the maximal constraint admissible set $\mathcal{X}_{M}=\{x \in \overline{\mathcal{X}}: x(0)=$ $x \Longrightarrow x(t) \in \overline{\mathcal{X}}, \forall t \in[0, \infty)\}$. Thus, $\mathcal{X}^{\infty} \supseteq \mathcal{X}_{M}$, and by maximality of $\mathcal{X}_{M}, \mathcal{X}_{M} \supseteq \mathcal{X}^{\infty}$, so that $\mathcal{X}_{M}=\mathcal{X}^{\infty}$. Finally, if $\overline{\mathcal{X}}$ is a polytope, then the constraints in (16) define a polytope with finite facets, since at any step $t$, a finite number of constraints is added.

\section{B. Stabilizing constrained MAPC with terminal set}

Next, we exploit the existence of the PI set within $\overline{\mathcal{X}}$ proved in Section IV-A to construct a terminal set $\mathcal{X}_{N}$ for the constrained MAPC. Due to the guaranteed existence of $\mathcal{X}^{\infty}$, this can be computed efficiently by backward reachability iterations [19].

Lemma 5: Consider (2) and $\kappa(\xi)=\sum_{i}\left[\xi_{i}\right] K_{i}$ as in Lemma 1. . Let $\mathcal{X}^{\infty}$ be a convex PI set for all $x(t+1)=$ $\left(A_{i}+B K_{i}\right) x(t), i \in \mathbb{Z}_{[1, \ell]}$. Then, $\mathcal{X}^{\infty}$ is PI for $x(t+1)=$ $\sum_{i=1}^{\ell}[\xi]_{i} A_{i} x(t)+B \kappa(\xi) x(t)$, for all $\xi \in \Xi$.

$\mathcal{X}^{\infty}$ in Lemma 5 can be interpreted as a PI set contained in $\overline{\mathcal{X}}$ for the pLDI (15), and it can be computed as follows.

Lemma 6: Consider (15) subject to constraints $x \in \mathcal{X}$, $u \in \mathcal{U}$, where $\mathcal{X}, \mathcal{U}$ are convex sets, and let $K_{i}, i \in \mathbb{Z}_{[1, \ell]}$ be designed as in Lemma 1. Let $\mathcal{X}^{(0)}=\left\{x \in \mathbb{R}^{n}: x \in\right.$ $\left.\mathcal{X}, K_{i} x \in \mathcal{U}, \forall i \in \mathbb{Z}_{[1, \ell]}\right\}$ and the set sequence $\left\{\mathcal{X}^{(h)}\right\}_{h=0}^{\infty}$ be such that

$$
\begin{aligned}
& \mathcal{X}^{(h+1)}=\left\{x \in \mathbb{R}^{n}:\left(A_{i}+B K_{i}\right) x \in \mathcal{X}^{(h)},\right. \\
&\left.\forall i \in \mathbb{Z}_{[1, \ell]}\right\} \cap \mathcal{X}^{(h)} .
\end{aligned}
$$

There exists $\bar{h} \in \mathbb{Z}_{0+}$ such that $\mathcal{X}^{(\bar{h})}=\mathcal{X}^{(\bar{h}+1)}, \mathcal{X}^{\infty}=\mathcal{X}^{(\bar{h})}$ is PI, and for all $x \in \mathcal{X}^{\infty}$ and for all $\xi \in \Xi$, it holds that $x \in \mathcal{X}, \kappa(\xi) x \in \mathcal{U}$.

The proof, omitted here due to limited space, is based on standard invariant set methods [19] combined with Theorem 2 which guarantees the existence of $\bar{h} \in \mathbb{Z}_{0+}$ such that if the constraints are satisfied for $\bar{h}$ steps, they are satisfied for any number of steps $h>\bar{h}$.

Theorem 3: Consider (2), (3), $K_{i}, P_{i}, i \in \mathbb{Z}_{[1, \ell]}$ be obtained as in Lemma 1, and let $\mathcal{X}^{\infty}$ be the PI set obtained as in Lemma 6. Let $\mathcal{X}_{N}=\mathcal{X}^{\infty}=\left\{x: H_{N} x \leq K_{N}\right\}$, and $P(\xi)=\sum \xi_{i} P_{i}$ in the MPC problem (4). Then, for every $\xi \in \Xi$, the origin of the closed-loop system is asymptotically stable in $\mathcal{X}_{f}=\{x \in \mathcal{X}:(4)$ feasible for $x(t)=x\}$.

Proof (sketch): The proof of Theorem 3 follows from the existence of $\mathcal{X}^{\infty}$ with non-empty interior by Theorem 2 using the standard arguments of the stability proofs for constrained MPC based on terminal cost and terminal set [9], and it is only sketched due to space limitations. The invariance of $\mathcal{X}_{f} \subseteq \mathcal{X}$ for the closed-loop system is proved by constructing a feasible input sequence as in Theorem 1, where now $\tilde{u}_{N-1 \mid t+1}=\kappa(\xi) x_{N-1 \mid t+1}$, and by exploiting that $x_{t \mid N} \in \mathcal{X}_{N}=\mathcal{X}^{\infty} \subseteq \overline{\mathcal{X}}$, that (9) is feasible
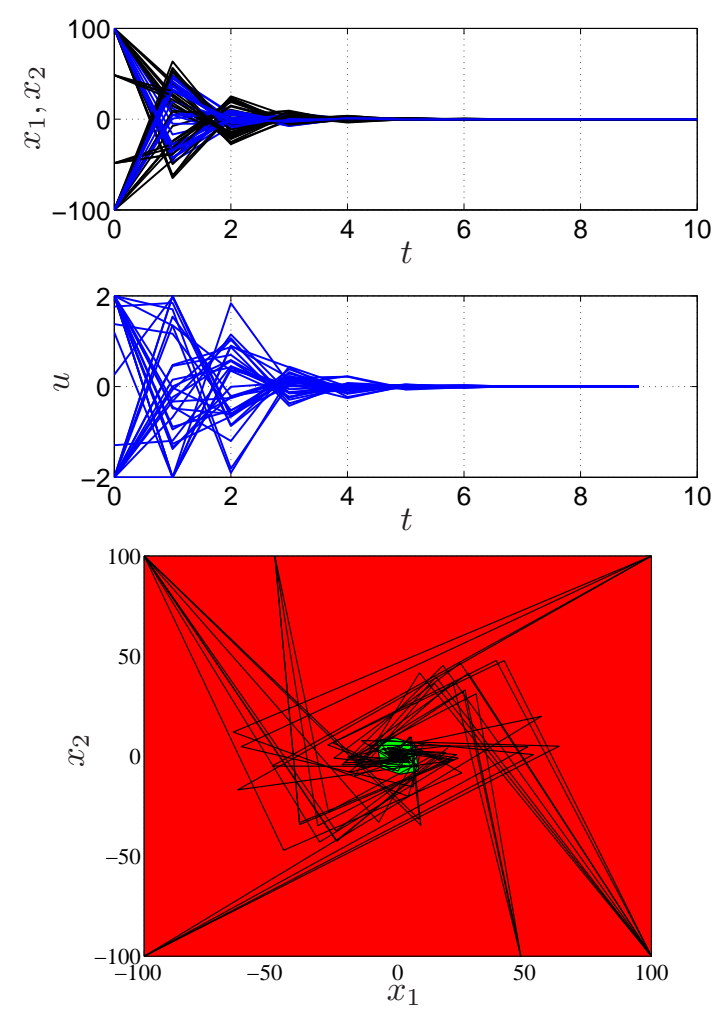

Fig. 1. MAPC for the case of $\ell=6$ random stable $2^{\text {nd }}$ order systems. Top: time trajectories, $x_{1}$ (blue), $x_{2}$ (black), $u$ (blue). Bottom: phase plane trajectories (black), $\mathcal{X}$ (red), $\mathcal{X}^{\infty}$ (green).

for $x_{N-1 \mid t+1} \in \mathcal{X}_{N}$, and that $\mathcal{X}_{N}$ is PI for (2) in closed-loop with (9), for all $\xi \in \Xi$. The rest of the proof follows using as Lyapunov function $\mathcal{V}_{\mathrm{MPC}}^{(\xi)}$ as in Theorem 1 , with $\mathcal{X}_{f}$ as the PI set.

\section{EXAMPLES}

First, we consider the case where $\ell=6$ and the vertex systems of (2) are second order systems with randomly generated AS state update matrices $A_{i}, i \in \mathbb{Z}_{[1, \ell]}$, with a single input and a common input to state matrix $B$. The system is subject to constraints $-100 \leq x \leq 100,-2 \leq u \leq$ 2 . In the MAPC we set $N=10, Q=0.1 \cdot I, R=1$. We design the terminal cost and auxiliary control law based on Lemma 1, and the terminal set based on Lemma 6 . We show the simulations for 8 initial conditions $x(0)=\operatorname{sat}\left(c_{0} v\right)$, where sat denotes saturation in the admissible state range, $v$ is randomly chosen in $\left\{v_{i}\right\}_{i=1}^{n_{v}}$, which is the set of vertices of $\mathcal{X}^{\infty}$, and $c_{0}=50$. For each initial condition, 4 different simulations are obtained by randomly choosing values of $\xi \in \Xi$. The results are shown in Figure 1 .

Next, we consider a case where $\ell=5, A_{1}=\left[\begin{array}{cc}1 & 0.2 \\ 0 & 1\end{array}\right]$, $A_{2}=1.1 \cdot A_{1}, A_{3}=0.9 \cdot A_{1}, A_{4}=\left[\begin{array}{ccc}0.9 & 0.3 \\ 0.4 & 0.6\end{array}\right] A_{5}=\left[\begin{array}{ccc}9.95 & 0 \\ 8 & 1.03\end{array}\right]$, and $B=[-0.035-0.905]$. Note that some of the vertex systems of (2) are stable and some are unstable. The system is subject to constraints $-40 \leq x \leq 40,-10 \leq u \leq 10$. In the MAPC we set $N=7, \bar{Q}=0.01 \cdot I, R=1$. Again, we show the simulations for 8 initial conditions chosen with 

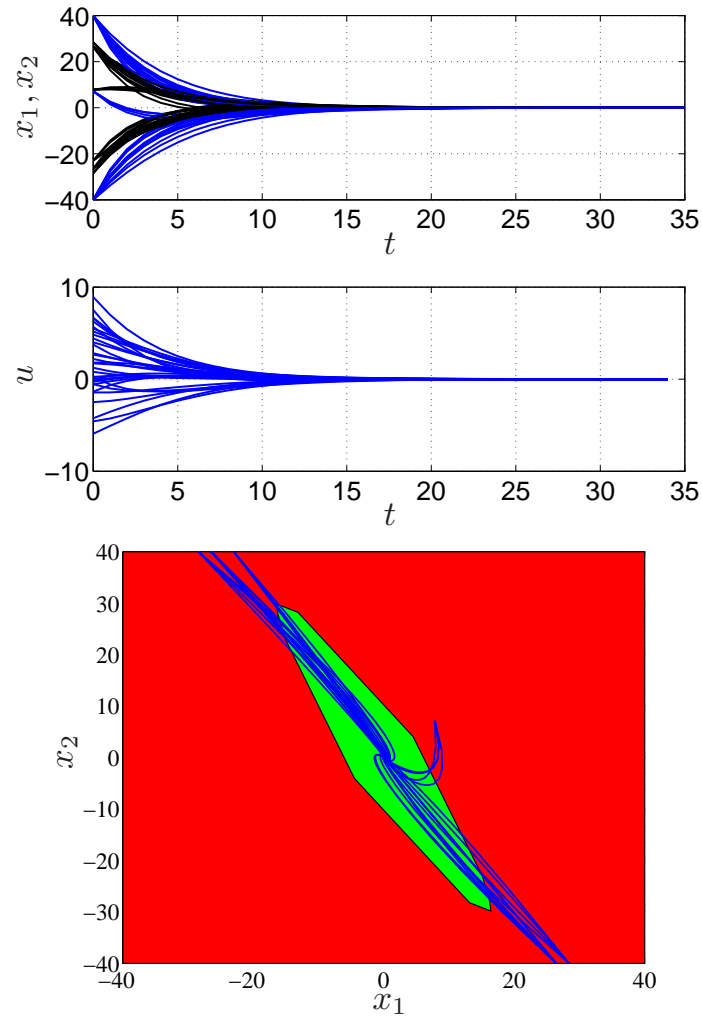

Fig. 2. MAPC for the case of $\ell=5$ stable and unstable $2^{\text {nd }}$ order systems. Top: time trajectories, $x_{1}$ (blue), $x_{2}$ (black), $u$ (blue). Bottom: phase plane trajectories (black), $\mathcal{X}$ (red), $\mathcal{X}^{\infty}$ (green).

the same method as in the previous test, with $c_{0}=2$, and for each initial condition, 4 different systems obtained by randomly choosing values of $\xi \in \Xi$. The results are reported in Figure 2, which shows again that the closed loop is AS.

For this case it can be shown that an MPC designed with the exact prediction model (i.e., with the correct value for $\xi \in \Xi$ in (4)) but with terminal cost designed based on a single vertex system, may fail to asymptotically stabilize the closed loop, and may actually result in a steady state error.

\section{Conclusions and Future Research}

We have proposed a model adjustable predictive control strategy that allows to retain stability guarantees with minimal computations after adjusting the prediction model. Thus, the method allows for fine tuning of the model after deployment in a microcontroller. The key idea is to use a parameter-dependent Lyapunov function to design the MAPC terminal cost and an auxiliary terminal control law, and to use the auxiliary control law to determine a constraint-admissible polyhedral invariant set that is used as MAPC terminal set. We proved the existence of such a terminal set with nonempty interior and asymptotic stability of the closed-loop.

Besides solving the problem of MAPC we believe that the proposed framework establishes the basis for an adaptive model predictive control with stability guarantees, where the adjustments to the prediction model parameters occur concurrently to the control, i.e., the convex combination vector is a time-varying parameter. Even if for MAPC it introduces conservativeness, the property that the pLF is guaranteed to decrease regardless of a change in the convex combination vector is of fundamental importance to this end. The development of such an adaptive model predictive control strategy is currently being investigated.

\section{REFERENCES}

[1] D. Hrovat, S. Di Cairano, H. E. Tseng, and I. V. Kolmanovsky, "The development of model predictive control in automotive industry: A survey," in IEEE Int. Conf. Control Applications, 2012, pp. 295-302.

[2] S. Di Cairano, "An industry perspective on MPC in large volumes applications: Potential Benefits and Open Challenges," in Proc. 4th IFAC Nonlinear Model Predictive Control Conference, Noordwijkerhout, The Netherlands, 2012, pp. 52-59.

[3] A. Bemporad, M. Morari, V. Dua, and E. Pistikopoulos, "The Explicit Linear Quadratic Regulator for Constrained Systems," Automatica, vol. 38 , no. 1, pp. 3-20, 2002.

[4] S. Richter, C. N. Jones, and M. Morari, "Computational complexity certification for real-time mpc with input constraints based on the fast gradient method," IEEE Trans. Automatic Control, vol. 57, no. 6, pp. 1391-1403, 2012

[5] S. Di Cairano, M. Brand, and S. A. Bortoff, "Projection-free parallel quadratic programming for linear model predictive control," Int. J. Control, vol. 86, no. 8, pp. 1367-1385, 2013.

[6] A. U. Raghunathan and S. Di Cairano, "Alternating direction method of multipliers for strictly convex quadratic programs: Optimal parameter selection," in Proc. American Contr. Conf. IEEE, 2014, pp. 4324-4329.

[7] S. Di Cairano and A. Bemporad, "Model predictive control tuning by controller matching," IEEE Trans. Automatic Control, vol. 55, no. 1, pp. 185-190, 2010.

[8] E. N. Hartley and J. M. Maciejowski, "Designing output-feedback predictive controllers by reverse-engineering existing LTI controllers," IEEE Trans. Automatic Control, vol. 58, no. 11, pp. 2934-2939, 2013.

[9] J. B. Rawlings and D. Q. Mayne, Model Predictive Control: Theory and Design. Nob Hill, Madison, Wisconsin, 2009, appendices available at http://jbrwww.che.wisc.edu/home/jbraw/mpc/webappendices.pdf.

[10] M. V. Kothare, V. Balakrishnan, and M. Morari, "Robust constrained model predictive control using linear matrix inequalities," Automatica, vol. 32 , no. 10 , pp. $1361-1379,1996$

[11] F. A. Cuzzola, J. C. Geromel, and M. Morari, "An improved approach for constrained robust model predictive control," Automatica, vol. 38 , no. 7 , pp. 1183-1189, 2002.

[12] M. C. de Oliveira, J. Bernussou, and J. C. Geromel, "A new discretetime robust stability condition," Systems \& control letters, vol. 37, no. 4, pp. 261-265, 1999.

[13] J. Daafouz and J. Bernussou, "Parameter dependent lyapunov functions for discrete time systems with time varying parametric uncertainties," Systems \& control letters, vol. 43, no. 5, pp. 355-359, 2001.

[14] W. H. Heemels, J. Daafouz, and G. Millerioux, "Observer-based control of discrete-time lpv systems with uncertain parameters," Automatic Control, IEEE Transactions on, vol. 55, no. 9, pp. 2130-2135, 2010.

[15] N. Wada, K. Saito, and M. Saeki, "Model predictive control for linear parameter varying systems using parameter dependent lyapunov function," Circuits and Systems II: Express Briefs, IEEE Transactions on, vol. 53, no. 12, pp. 1446-1450, Dec 2006.

[16] S. Yu, C. Bohm, H. Chen, and F. Allgower, "Stabilizing model predictive control for lpv systems subject to constraints with parameterdependent control law," in Proc. American Contr. Conf. IEEE, 2009, pp. $3118-3123$.

[17] E. Gilbert and K. Tan, "Linear systems with state and control constraints: the theory and applications of maximal output admissible sets," IEEE Trans. Automatic Control, vol. 36, no. 9, pp. 1008-1020, 1991

[18] M. Lazar, "Model predictive control of hybrid systems: Stability and robustness," Ph.D. dissertation, Eindhoven University of Technology, The Netherlands, 2006

[19] F. Blanchini and S. Miani, Set-theoretic methods in control. Springer Science \& Business Media, 2007. 